\title{
Time-Varying Characteristics of the Longitudinal Vibration of a High-Speed Traction Elevator Lifting System
}

\author{
Qing Zhang \\ School of Mechanical Engineering,Tianjin University, Tianjin City, China. \\ School of Mechanical and Electrical Engineering, Shandong Jianzhu University, Jinan City, China. \\ Tao Hou, Rui-Jun Zhang and Jie Liu \\ School of Mechanical and Electrical Engineering, Shandong Jianzhu University, Jinan City, China.
}

(Received 19 June 2018; accepted 22 November 2018)

\begin{abstract}
Hoisting rope in a high-speed traction elevator lifting system exhibits strong time-varying characteristics, which, to a large extent, affect the comfort and safety of high-speed elevators. In order to analyse the influence of the time-varying characteristics on the vibration of elevators during the whole operation process, the longitudinal vibration of the hoisting rope of a lifting elevator is introduced to study the effect of the time varying characteristics of a lifting system on the time-varying characteristics of longitudinal vibration. The nonlinear time-varying model of the longitudinal vibration of the hoisting rope is established using the Hamilton principle and energy method. The Galerkin method is used to discretize the partial differential equations of vibration. The quintic polynomial is used to fit the ideal operating state of the elevator. Following this, the quintic polynomial is input as the motion parameter. The precise integration for the time-varying model of longitudinal vibration of the lift system is put forward. The time variant of elevator hoisting system is solved and vibration analysis is carried out with elevator case. It is observed that, during operation, as the length of the hoist rope increases, the acceleration of the longitudinal vibration of the elevator increases too. As the tensile force, provided by the tensioning device, increases, the acceleration decreases.
\end{abstract}

\section{INTRODUCTION}

The continuous emergence of high-rise and super high-rise buildings prompted the development of high-trip, high-speed elevators. In the course of operation, elevators inevitably experience a variety of vibration phenomena, with a large part of vibration being related to the elevator lifting system.

An elevator lift system is mainly composed of hoisting ropes with a certain axial movement length, a counterweight at one end, and a car at the other end. Hoisting ropes exhibit certain time-varying characteristics. As the lifting part of the elevator, hoisting ropes are elongated and shortened during elevator operation. Thus, parameters, such as stiffness and hoisting rope damping, constantly change. Furthermore, time-varying characteristics are apparent at high speed, thereby seriously affecting the comfort and safety of elevators. With the change in rope length, natural frequencies and vibration energy also change. When the rope is shortened, free vibration energy increases exponentially, and dynamic instability easily occurs, ${ }^{1}$ thereby seriously affecting the safety of passengers. The effect of longitudinal vibration on the lifting system is markedly stronger than that of lateral vibration under consistent initial conditions. $^{2}$ Therefore, the effect of the time-varying characteristics of hoisting ropes, in the longitudinal vibration of the elevator lifting system, should be studied.

Numerous studies on the longitudinal vibration of traction elevator hoisting systems have been conducted. ${ }^{3-9}$ The basis of these studies is to establish discrete models based on distributed parameters, in which the wire rope in the hoisting system is separated into a number of time-varying characteristics of quality-spring-damping systems. Parameters vary with changes of the elevator operating state. The advantage of this type of model is that the established ordinary differential equations are easy to understand and solve. However, this type of model disregards the continuity of wire rope flexibility and thus cannot effectively reflect the dynamics of elevator lifting systems. Therefore, the continuous model based on a distributed parameter, which can describe the flexible characteristics of traction ropes better, is gradually applied.

The distributed parameter continuous system model generally simplifies the lifting system to an axially moving chord with a certain mass attached at one end, and the kinetic behavior of the system is described by establishing either a functional differential control or a partial differential control equation. The parameters of the model are continuously distributed in time and space; thus, the model possesses infinite degrees of freedom and can satisfactorily describe the flexible continuous characteristics of the elevator lifting system. Zhang established differential equation of motion and energy equations representing the longitudinal vibration of an arbitrary variable-length flexible lifting system by using the Hamilton principle and validated the accuracy of continuous system modeling and energy methods. ${ }^{10}$ Bao established the equations governing flexible lifting wire ropes by using the Hamilton principle, studied the nonlinear vibration of a flexible lifting wire rope with timevarying length, and verified the theoretical model by experiment. ${ }^{11,12}$ In addition, considering the interaction between the rigid and the deformation motions of the hoisting rope, Bao established a differential equation that represented the longitudinal vibration of the hoisting rope of a variable-length lifting system, and a numerical example is given to analyse the model. ${ }^{13}$ These studies considered the high-speed elevator lifting system as the research object. However, the weight of the 
compensation rope and the tensile force provided by the tensioning device are not considered when the model is developed. The weight of the compensation rope and tensile force are the existing objectives in high-speed elevators. Therefore, the established model is reasonable when the weight of the compensating rope and the tensile force provided by the tensioning device are considered.

The exact solution for the time-varying model of the longitudinal vibration of a high-speed elevator hoisting system cannot be easily obtained; thus, a numerical solution is usually applied. In literature, the Runge-Kutta method was used to solve the model, but the algorithm poses a computational stability problem and requires a small step size. ${ }^{13}$ Therefore, the number of steps and the amount of calculation increases, which can in turn result in error accumulation. Precise integration is an effective numerical calculation method proposed by Prof. Zhong. ${ }^{14}$ This method offers the most important features of high precision, show its absolute stability, and is widely used in solving linear stationary systems. At present, literature successfully applied a fine integral method to evaluate the transient response of time-varying systems. ${ }^{1-15}$

We established the control equation corresponding to the longitudinal vibration of the hoisting rope in a high-speed traction elevator lift system. This approach was based on the Hamilton principle and considered the weight of the compensating rope and the tensile force provided by the tensioning device. The Galerkin weighted residual method was used for discretization. The time-domain curves of the longitudinal vibration response of the hoisting rope were simulated by the fine integral method. The dynamic characteristics of the elevator, influence of car mass and hoisting rope mass, and tension on the longitudinal vibration of the elevator are analysed during the upward and downward process.

\section{NONLINEAR TIME-VARYING MODEL OF THE LONGITUDINAL VIBRATION OF HOISTING ROPES IN A HIGH SPEED TRACTION ELEVATOR SYSTEM}

To study the time-varying characteristics of the longitudinal vibration of traction rope conveniently, the modeling and solving of this paper were based on the following three assumptions:

(1) Hoisting ropes are continuous and uniform, with constant cross-sectional area $A$ and elastic modulus $E$ during movement;

(2) The influence of lateral vibration from the hoisting ropes is ignored, and elastic deformation caused by the vertical vibration is neglectable with respect to the length of the ropes.

(3) The influence of bending rigidity on hoisting ropes, friction force, and airflow are ignored.

Figure 1 shows a diagram of a hoisting system for a highspeed traction elevator. Considering the influence of the time variation of the traction rope, the compensating rope and the tensioning device on the longitudinal vibration, the elevator vibration system was regarded as consisting of a traction rope, a car and a compensation rope. Thus, Fig. 1 was simplified into a longitudinal vibration time-varying model of the traction rope in a elevator hoisting system, as shown in Fig. 2.

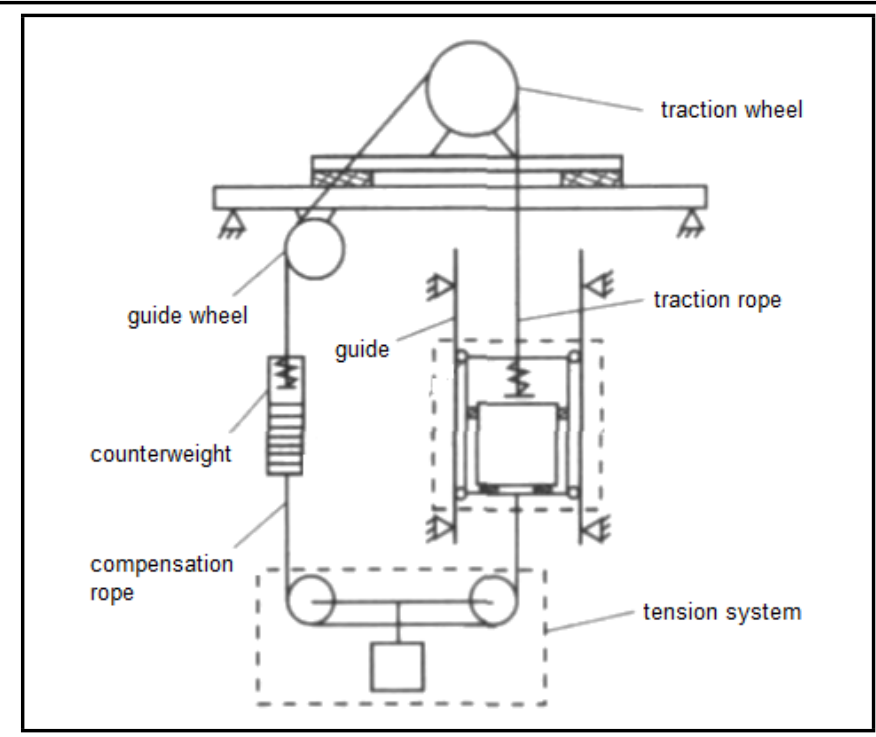

Figure 1. Structure of an elevator lifting system.

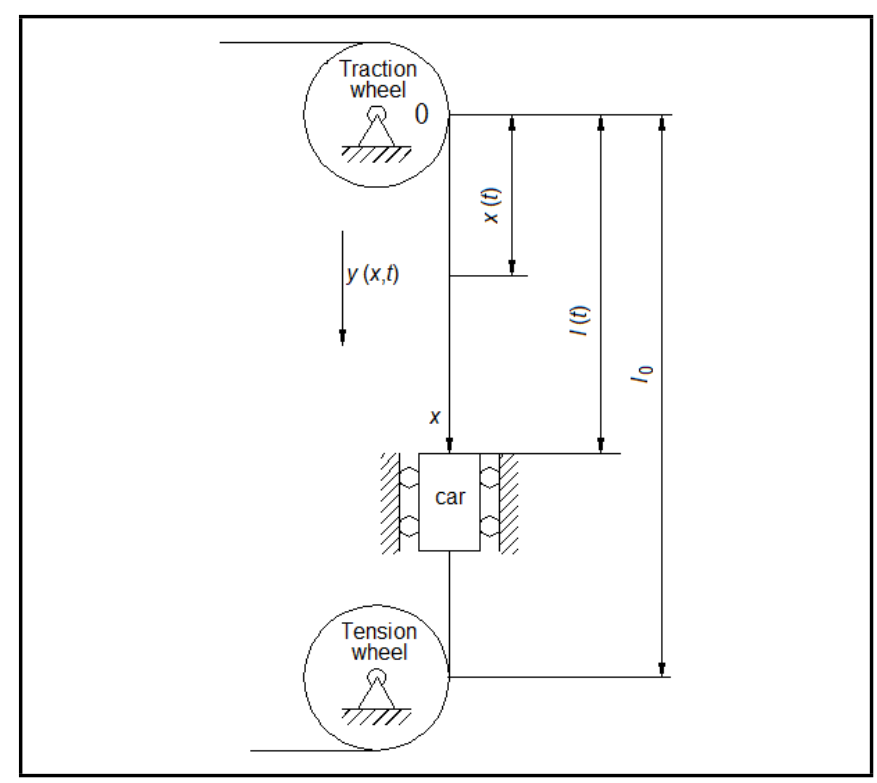

Figure 2. Time-varying model of the hoisting rope in an elevator lifting system.

\subsection{Dynamic Equations of a High-Speed Traction Elevator Hoisting System}

Figure 2 shows the time-varying model of longitudinal vibration in a high-speed traction elevator lifting system. The hoisting rope of the high-speed traction elevator was simplified as a variable-length string along the axial force and movement. The particular structure of the car was ignored and simplified into a rigid weight block of mass $m$ connected to the lower end of the cord. $\rho_{1}$ was the density of the hoisting rope, $A$ was the cross-sectional area, $E$ was the elastic modulus, and $\rho_{2}$ was the compensation rope density. The traction wheel was tangential to the rope, and the positive sense of $x$-axis was vertically downwards. The hoisting rope length at the top of the car from the origin of the coordinate was $l(t)$. Displacement at the position of the cord $x(t)$ was $y(x(t), t)$ and $v(t)$ for a high-speed traction elevator at the operating speed. $l_{0}$ was the maximum lift height (high-speed traction elevators are generally used for super high-rise buildings; in addition, the car height relative to the high-rise building is extremely small; therefore, car height can be ignored). 
By using the finite deformation theory of continuum, the displacement vector and velocity vector of $v(t)$ in the $x$-axis were as follows:

$$
\begin{aligned}
\mathbf{r} & =[x(t)+y(x(t), t)] \mathbf{j} \\
\mathbf{V} & =\left[v(t)+y_{t}(x(t), t)\right] \mathbf{j}
\end{aligned}
$$

where $\mathbf{j}$ was the unit vector in the $x$-axis direction, $y_{t}(x(t), t)$ is the derivative of $y(x(t), t)$ with respect to $t$, and $y, y_{t}$ represented $y(x(t), t)$ and $y_{t}(x(t), t)$, respectively.

Similarly, the displacement vector and velocity vector of the car in the $x$-axis direction were as follows:

$$
\begin{aligned}
\mathbf{r}_{c} & =[l(t)+y] \mathbf{j} \\
\mathbf{V}_{c} & =\left[v(t)+y_{t}\right] \mathbf{j} .
\end{aligned}
$$

The kinetic energy of the system was expressed as follows:

$$
E_{k}=\left.\frac{1}{2} m V^{2}\right|_{x=l(t)}+\frac{1}{2} \rho_{1} \int_{0}^{l(t)} V^{2} d s .
$$

The elastic potential of the system was:

$$
E_{s}=\int_{0}^{l(t)}\left(P y_{x}+\frac{1}{2} E A y_{x}^{2}\right) d s
$$

where $y_{x}(x(t), t)$ was the derivative of $y(x(t), t)$ with respect to $x$, and $y_{x}$ represented $y_{x}(x(t), t) . P$ was the tensile force of the rope during a static balance of tension. With its own gravity and that of the car, the hoisting rope was subjected to the tension of the tensioned rope and the tensile force applied by the tensioning device. Thus, tension in the static equilibrium was expressed as:

$$
P=\left[m+\rho_{1}(l(t)-x)+\rho_{2}\left(l_{0}-l(t)\right)\right] g+f .
$$

The gravitational potential energy of the system was expressed as:

$$
E_{g}=-\int_{0}^{l(t)} \rho_{1} g d t-\left.m g y\right|_{x=l(t)} .
$$

According to the Hamilton principle:

$$
I=\int_{t_{1}}^{t_{2}}\left[\delta E_{k}-\delta E_{s}-\delta E_{g}\right] d t=0 .
$$

The use of time and geometric boundary conditions yielded the following:

$$
\delta y(0, t)=\delta y\left(x, t_{1}\right)=\delta y\left(x, t_{2}\right)=0 .
$$

The derivation law of the parametric integral and partial integration method was applied as follows:

$$
\begin{aligned}
\frac{\partial}{\partial t} \int_{0}^{l(t)} \rho\left(v+y^{\prime}\right) \delta y d x= & \int_{0}^{l(t)} \rho_{1}\left(v+y^{\prime}\right) \delta \dot{y} d x+ \\
& \int_{0}^{l(t)} \frac{\partial}{\partial t} \rho_{1}\left(v+y^{\prime}\right) \delta y d x+\left.\rho_{1}\left(v+y^{\prime}\right) \delta y\right|_{x=l(t)}
\end{aligned}
$$

and deducted from the following:

$$
\begin{array}{r}
\int_{t_{1}}^{t_{2}} \int_{0}^{l(t)}\left[\rho_{1}\left(y_{t t}+a\right)-P_{x}-\rho_{1} g-E A y_{x x}\right] \delta y d x d t+ \\
\int_{t_{1}}^{t_{2}}\left[m\left(a+y_{t t}\right)+\rho_{1} v\left(v+y_{t}\right)-m g+P+E A y_{x}\right] \\
\left.\delta y\right|_{x=l(t)} d t=0
\end{array}
$$

The longitudinal vibration of the high-speed traction elevator lifting system was obtained using the dynamic equation as follows:

$$
\begin{gathered}
\rho_{1}\left(y_{t t}+a\right)-P_{x}-\rho_{1} g-E A y_{x x}=0, \quad 0<x<l(t) ; \\
m\left(a+y_{t t}\right)+\rho_{1} v\left(v+y_{t}\right)+E A y_{x}+P-m g=0 \\
x=l(t)
\end{gathered}
$$

Equation (12) is the boundary condition of the cord at $x=l(t)$.

\subsection{Galerkin Discretization of the Time- Varying Partial Differential Equation for the Longitudinal Vibration of the Lift System}

The parameters of the partial differential control Eq. (11) with infinite degrees of freedom present strong time-varying characteristics. As a result, it was difficult to get their analytical solution. The coefficient matrix of the algebraic equations obtained by the Galerkin discrete method was symmetric and the approximation accuracy was higher than those of the other methods. Therefore, the Galerkin method was used to discretize the partial differential control equation. Then, differential equations with infinite degrees of freedom were discretized into ordinary differential equations with finite degrees of freedom and solved by numerical methods. For facilitating the discrete method, a dimensionless parameter $\xi$ was introduced and the original variables were normalized, which was $\xi=x / l(t)$. The time domain of $x$ became the fixed domain $[0,1]$ of $\xi$. Assuming that distribution function $y$ (with infinite degrees of freedom) can be used to represent the solution of Eq. (11):

$$
y(x, t)=\sum_{i=1}^{n} \varphi_{i}(\xi) q_{i}(t)=\sum_{i=1}^{n} \varphi_{i}\left(\frac{x}{l(t)}\right) q_{i}(t) .
$$

$\varphi_{i}(\xi)$ was the trial function, and $q_{i}(t)$ was the time-dependent generalized coordinates

$$
\varphi_{i}(\xi)=\sqrt{2} \sin \left(\frac{2 i-1}{2} \pi \xi\right), \quad(i=1,2, \ldots, n) .
$$

Then

$$
\begin{aligned}
y_{x}= & \frac{1}{l(t)} \sum_{i=1}^{n} \varphi_{i}^{\prime}(\xi) q_{i}(t) \\
y_{x x}= & \frac{1}{l(t)} \sum_{i=1}^{n} \varphi_{i}^{\prime \prime}(\xi) q_{i}(t) \\
y_{t}= & \sum_{i=1}^{n} \varphi_{i}(\xi) \dot{q}_{i}(t)-\frac{\xi v}{l(t)} \sum_{i=1}^{n} \varphi_{i}^{\prime}(\xi) q_{i}(t) ; \\
y_{t t}= & \sum_{i=1}^{n} \varphi_{i}(\xi) \ddot{q}_{i}(t)-\frac{2 \xi v}{l(t)} \sum_{i=1}^{n} \varphi_{i}^{\prime}(\xi) \dot{q}_{i}(t)+ \\
& \frac{2 \xi v^{2}}{l^{2}(t)} \sum_{i=1}^{n} \varphi_{i}^{\prime}(\xi) q_{i}(t)-\frac{a \xi}{l(t)} \sum_{i=1}^{n} \varphi_{i}^{\prime}(\xi) q_{i}(t)+ \\
& \frac{\xi^{2} v^{2}}{l^{2}(t)} \sum_{i=1}^{n} \varphi_{i}^{\prime \prime}(\xi) q_{i}(t) .
\end{aligned}
$$

Equation (15) was substituted into the kinetic Eq. (12). Both sides were multiplied by $\varphi_{j}(\xi)$, and $\xi$ was integrated in the 
range $[0,1]$. Boundary condition Eq. (13) was applied to Eq. (15), and the two sides were multiplied by $\varphi_{j}(1)$ after transformation. The original partial differential equations were discretized into the following equation by using the weighted residual method:

$$
\mathbf{M} \ddot{\mathbf{q}}_{j}+\mathbf{C} \dot{\mathbf{q}}_{j}+\mathbf{K} \mathbf{q}_{j}=\mathbf{F} ;
$$

where $\mathbf{q}_{j}=\left[q_{1}(t), q_{2}(t), \ldots, q_{n}(t)\right]$ was the generalized coordinate vector,

$$
\begin{aligned}
\mathbf{M}= & \rho_{1} \delta_{i j}+\frac{m}{l} \varphi_{i}(1) \varphi_{j}(1) ; \\
\mathbf{C}= & -\frac{2 \rho_{1} v}{l} \int_{0}^{1} \xi \varphi_{i}^{\prime} \varphi_{j} d \xi+\frac{\rho_{1} v}{l} \varphi_{i}(1) \varphi_{j}(1) ; \\
\mathbf{K}= & \frac{m v^{2}}{l^{3}} \varphi_{i}^{\prime \prime}(1) \varphi_{j}(1)-\frac{\rho_{1} a}{l} \int_{0}^{1} \xi \varphi_{i}^{\prime} \varphi_{j} d \xi- \\
& \frac{\rho_{1} v^{2}}{l^{2}} \int_{0}^{1} \xi^{2} \varphi_{i}^{\prime} \varphi_{j}^{\prime} d \xi-\frac{E A}{l^{2}} \int_{0}^{1} \varphi_{i}^{\prime \prime} \varphi_{j} d \xi ; \\
\mathbf{F}= & -\rho_{1} a \int_{0}^{1} \varphi_{j} d \xi-\frac{m a}{l} \varphi_{j}(1)-\frac{\rho_{1} v}{l} \varphi_{j}(1)- \\
& \frac{\rho_{2} g\left(l_{0}-l\right)+f}{l} \varphi_{j}(1) .
\end{aligned}
$$

\section{PRECISE INTEGRATION FOR THE TIME VARYING MODEL OF LONGITUDINAL VIBRATION OF THE LIFT SYSTEM}

For the solution of the $n$-dimensional nonlinear timevarying differential equation shown in Eq. (16), the general numerical method cannot achieve high accuracy because of its time-varying characteristics. Precise integration was used to solve the problem and achieve highly accurate results.

First, follow the introduction of the dual variable of Hamiltonian system. Make

$$
p=M \dot{x}+C(t) x / 2 \quad \text { or } \quad \dot{x}=M^{-1} p-M^{-1} C(t) x / 2 .
$$

By substituting the above formula into the dynamic equation, the following equation can be obtained:

$$
\dot{p}=\left(C(t) M^{-1} C(t) / 4-K(t)\right) x-C(t) M^{-1} p / 2+f(t) .
$$

The above equations were written in the general form of a linear system

$$
\left\{\begin{array}{l}
\dot{x}=A x+C p+r_{x} \\
\dot{p}=B x+D p+r_{p}
\end{array} ;\right.
$$

where: $A=M^{-1} C(t) / 2, B=C(t) M^{-1} C(t) / 4-K(t)$, $C=-C(t) M^{-1} / 2, D=M^{-1}, r_{p}=0, r_{x}=f(t)$.

Therefore,

$$
\dot{z}=H z+\phi(t)
$$

where: $z=\left[\begin{array}{l}x \\ p\end{array}\right], H=\left[\begin{array}{ll}A & B \\ C & D\end{array}\right], \phi(t)=\left[\begin{array}{l}r_{x} \\ r_{p}\end{array}\right]$.

Given that the non-homogeneous term is linear in the time step $\left(t_{k}, t_{k+1}\right)$, the equation was

$$
\dot{z}=H z+\phi_{k}+\dot{\phi}_{k}\left(t-t_{k}\right) .
$$

Then, the solution at $t_{k+1}$ moment was written as

$$
\begin{aligned}
z_{k+1}= & T_{k}\left[z_{k}+H_{k}^{-1}\left(\phi_{k}+H_{k}^{-1} \dot{\phi}_{k}\right)\right]- \\
& H_{k}^{-1}\left[\phi_{k}+H_{k}^{-1} \dot{\phi}_{k}+\dot{\phi}_{k}\left(t_{k+1}-t_{k}\right)\right] ;
\end{aligned}
$$

Table 1. Elevator movement profile regions.

\begin{tabular}{|c|c|c|}
\hline \hline Stage & Time required & Stage description \\
\hline 1 & $t_{j}$ & Acceleration increases to $a=-a_{\max }$ \\
2 & $t_{a}$ & Acceleration remains $-a_{\max }$ constant \\
3 & $t_{j}$ & Acceleration decreases to zero, $v=-v_{\max }$ \\
4 & $t_{v}$ & Speed $v_{\max }$ is unchanged \\
5 & $t_{j}$ & Acceleration increases to $a=a_{\max }$ \\
6 & $t_{a}$ & Acceleration remains $a_{\max }$ constant \\
7 & $t_{j}$ & Acceleration decreases to zero, $v=0$ \\
\hline
\end{tabular}

where $T_{k}=e^{H_{k}\left(t_{k+1}-t_{k}\right)}$. According to the given initial conditions of $z_{0}$ and with a constant step size $t_{k+1}-t_{k}$, the steps were gradually performed to obtain $z_{1}, z_{2}, \ldots, z_{k}, \ldots$ by Eq. (22), which is a typical "self-starting" algorithm.

\section{DESCRIPTION OF THE PARAMETERS FOR THE LONGITUDINAL VIBRATION DYNAMIC EQUATION}

The longitudinal vibration control Eq. (16) of the lift system of high-speed elevators showed that the structural parameters (hoisting weight, hoisting rope density, drag rope elastic modulus, hoisting rope cross-sectional area, and compensating rope density) and the operation state parameters (travel, speed, acceleration, and acceleration) of the elevator in the simulation and analysis of the dynamic equation of the high-speed elevator hoisting system were necessary inputs. In theoretical analysis, parameter values cannot be determined arbitrarily. Proper parameter selection was the premise of the dynamic behavior analysis of the lifting system and the key to reflect the vibration characteristics of the lift system.

\subsection{Elastic Modulus of the Hoisting Rope}

The values of elastic modulus given in current literature were somewhat different. Feyree experimentally measured the elastic modulus of a hoisting rope and recorded $8.3 \sim$ $12.5 \times 10^{10} \mathrm{~N} / \mathrm{m}^{2} .{ }^{16}$ The elevator lifting system included a rope spring and a car bottom vibration rubber, and the stiffness of these parts was less than that of the rope. Therefore, the elastic modulus of the hoisting rope was calculated in this study as $E=8 \times 10^{10} \mathrm{~N} / \mathrm{m}^{2}$.

\subsection{High-Speed Elevator Operating Status Parameters}

Because this article studied high-speed passenger elevator, high-speed and super high-speed elevators often only run in two specific floors. There was no stopping in the whole operation process. Therefore, the high-speed elevator operating state parameters here were the whole process of operation parameters during the upward and downward process.

The elevator uplink process was divided into seven stages according to the ideal operating curve of the elevator. Table 1 describes the curve phases of the lift operation and the operating curve of the elevator in each stage by fitting the ideal operating state into a quintic polynomial Eq. (23)

$$
l_{i}(t)=C_{0}^{i}+C_{1}^{i} t+C_{2}^{i} t^{2}+C_{3}^{i} t^{3}+C_{4}^{i} t^{4}+C_{5}^{i} t^{5} .
$$

\section{CASE ANALYSIS}

A high-speed elevator in Shandong (FUJIZY Elevator Co., Ltd.) was used as the case study. The properties of the elevator are as follows: hoisting rope density, $\rho_{1}=0.87 \mathrm{~kg} / \mathrm{m}$; crosssectional area, $A=89.344 \mathrm{~mm}^{2}$; compensation rope density, 


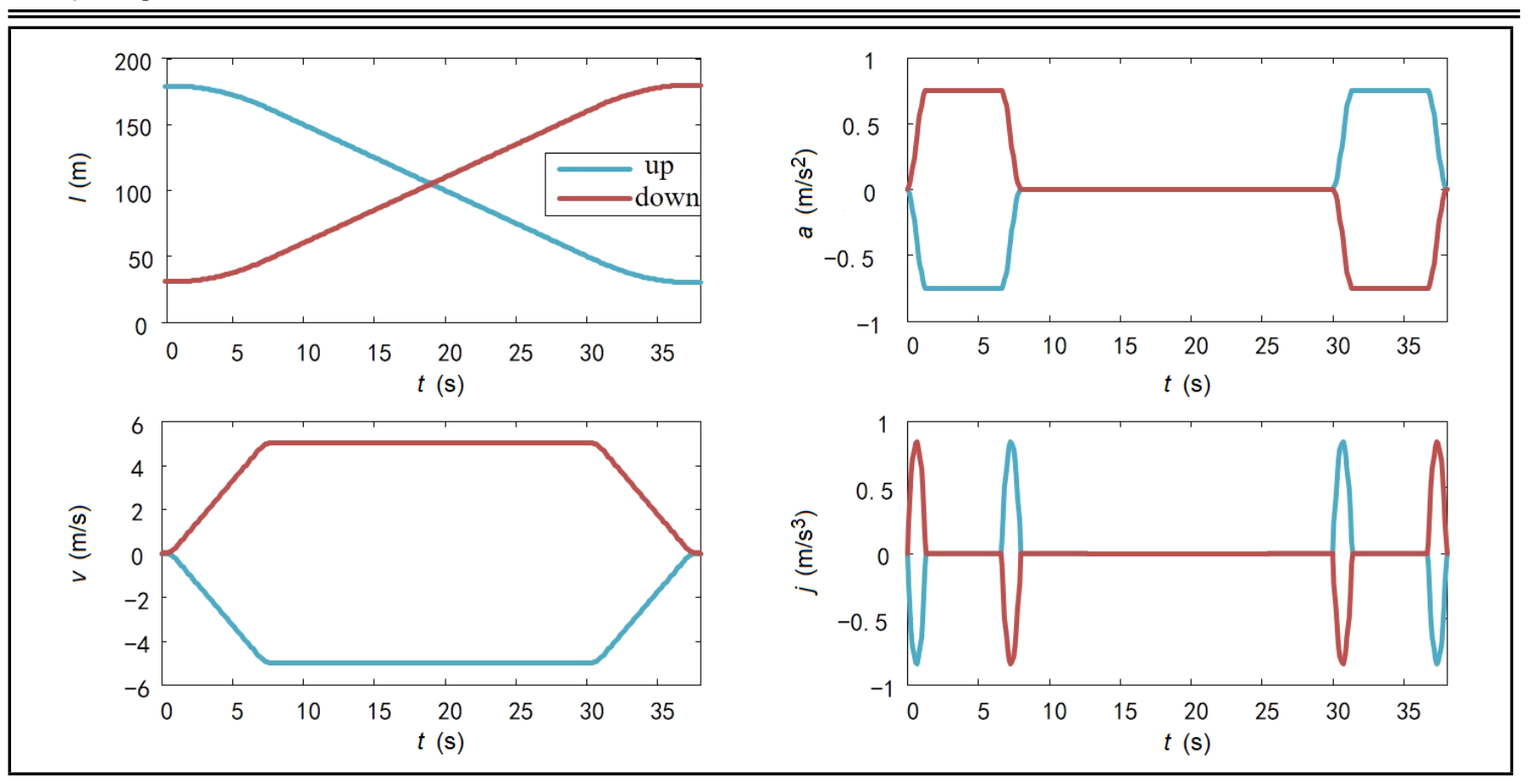

Figure 3. Curve of the movement profile of the elevator.

$\rho_{2}=0.343 \mathrm{~kg} / \mathrm{m}$; lifting quality of the single hoisting rope (including car, car frame, and rated load), $m=350 \sim 500 \mathrm{~kg}$; maximum speed, $5 \mathrm{~m} / \mathrm{s}$; maximum acceleration, $0.75 \mathrm{~m} / \mathrm{s}^{2}$; and rise height, $150 \mathrm{~m}$. Figure 3 shows the ideal operating curve of the elevator. Compared with other parameters in the time-varying dynamic Eq. (16) of the high-speed elevator hoisting system, such as density and elastic modulus, it is easier to adjust the lifting quality and tension force in actual production, and in today's elevator manufacturing and installation, the lifting quality and tension are usually adjusted, so the lifting quality and tension are adjusted. Therefore, the analysis and research on the influence of tension force is of great practical significance in the manufacture and installation of elevators. Combined with the analysis of time-varying influence of lifting system, the analysis in this paper can be divided into three parts: the dynamic characteristics of the elevator longitudinal vibration during the upward and downward process; the influence of tension on the longitudinal vibration of the elevator under tensile forces of 300, 400, and $500 \mathrm{~N}$; and the effect of single hoisting rope lifting quality (car, car frame, and rated load) on the longitudinal vibration of the elevator.

\subsection{Dynamic Characteristics of the Longitudinal Vibration During Upward and Downward Motion of the Elevator}

Given the hoisting quality of a single hoisting rope of $m=$ $400 \mathrm{~kg}$ and tensile force of $F=300 \mathrm{~N}$, the dynamic characteristics of the elevator upward and downward processes are analysed.

Figure 4 shows the dynamic characteristics of the elevator during the uplink process. The length of elevator hoisting rope gradually decreases as the elevator rises. However, the acceleration peak of the longitudinal vibration response gradually increases. The response displacement of the longitudinal vibration of the elevator repeatedly mutates during the upward process. It can be found from Fig. 4 that, during the upward process, the longitudinal vibration displacement of the high-speed elevator changes suddenly when the elevator runs $0-1.3 \mathrm{~s}$,
$6.6-7.9$ s, $29.9-31.2$ s, $36.7-38$ s. Comparing with the upward acceleration curve of the high-speed elevator in Fig. 3, it is found that the abrupt change in time for the elevator vibration displacement coincides with the change in time for the elevator acceleration. Based on this, it can be concluded that the longitudinal vibration is closely related to the acceleration of operation. Therefore, in the installation process of highspeed elevator, under the condition of meeting the design requirements, the elevator comfort can be improved by reducing the tension of the elevator.

Figure 5 shows the dynamic characteristics of the elevator during the downward process. The length of the elevator hoisting rope gradually increases with the downward movement of the elevator. The acceleration peak of longitudinal vibration response gradually decreases and the elevator longitudinal vibration gradually weakens. As shown in Fig. 5, during the downward process, the longitudinal vibration displacement of the high-speed elevator changes abruptly when the elevator runs $0-1.3 \mathrm{~s}, 6.6-7.9 \mathrm{~s}, 29.9-31.2 \mathrm{~s}$ and $36.7-38 \mathrm{~s}$. Compared with the downward acceleration curve of the high-speed elevator in Fig. 3, it can be determined that the longitudinal vibration of the elevator is closely related to the change of its running acceleration.

\subsection{Effect of Tensile Force $F$ on the Longitudinal Vibration of the Elevator}

With the hoisting of a single hoisting rope of $m=400 \mathrm{~kg}$, the influence of tensile force $F$ on the longitudinal vibrations of the elevator is analysed.

Figures 6(a), 6(b), and 6(c) show the acceleration responses of longitudinal vibration during the elevator upward and downward processes with tensile forces of $F=300,400$, and $500 \mathrm{~N}$. The response of longitudinal vibration acceleration of the upstream and downstream processes in Figs. 6(a), 6(b), and 6(c) is analysed. With the increasing tensile force of the tensioning system, the amplitude of the acceleration response increases. 


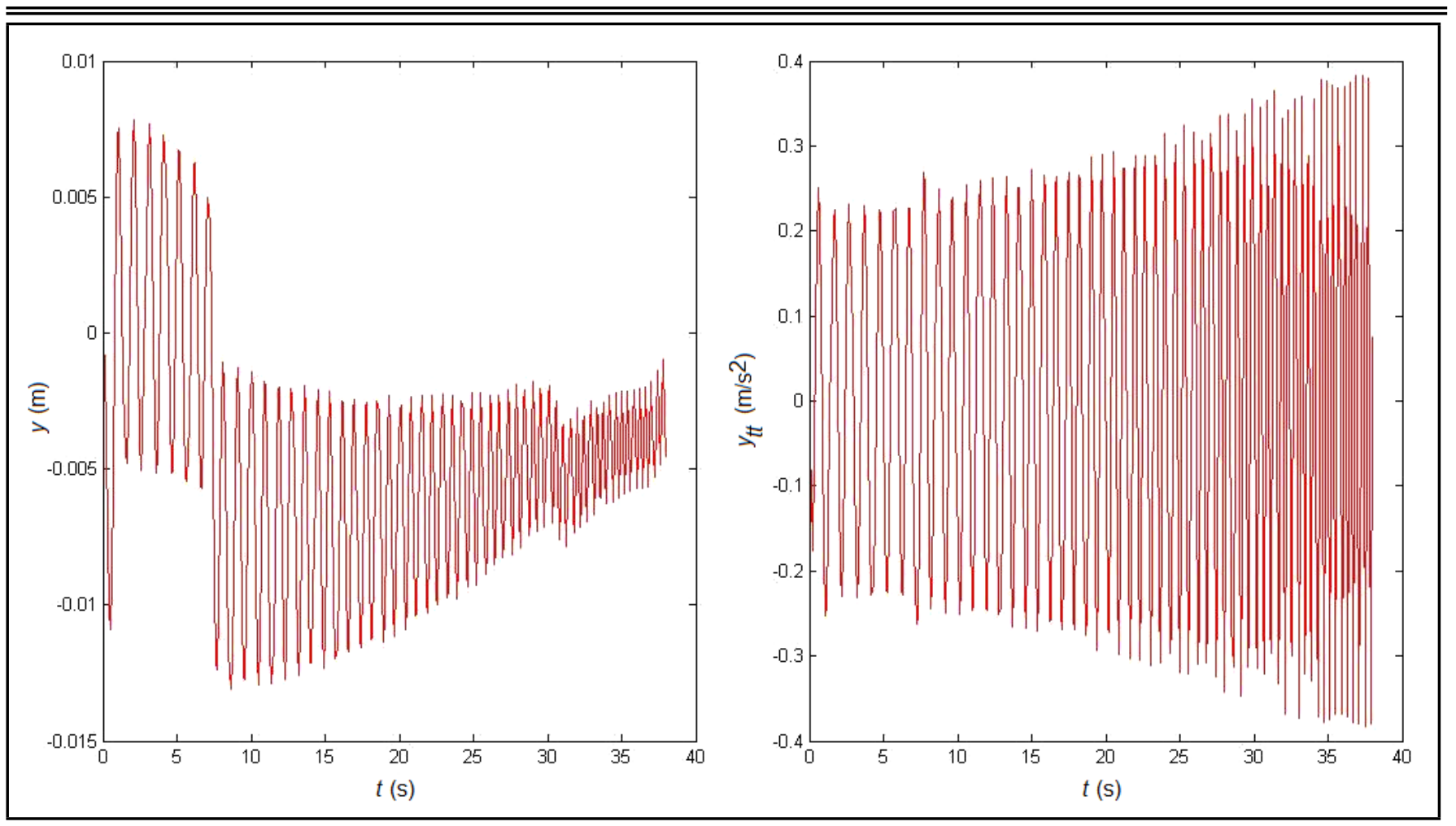

Figure 4. Dynamic characteristics of the elevator during the upward process.
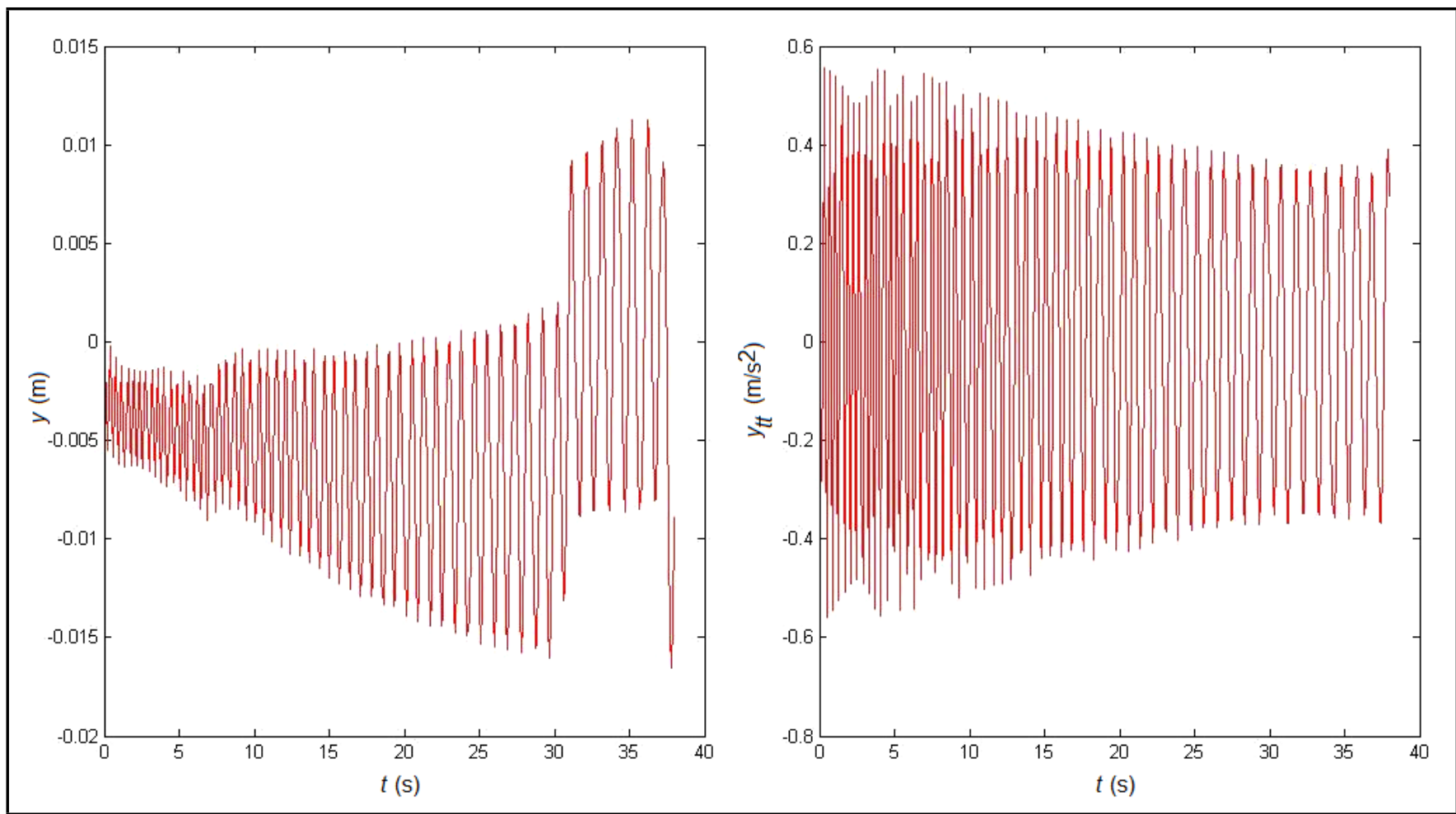

Figure 5. Dynamic characteristics of the elevator during the downward process.

\subsection{Influence of the Lifting Quality (Including Car, Car Frame, and Rated Load) of a Single Hoisting Rope on the Longitudinal Vibration of the Elevator}

A tensile force of $F=300 \mathrm{~N}$ of the tension system is considered; then, the dynamic characteristics of longitudinal vibration with the lifting quality values of a single hoisting rope of 350,400 , and $450 \mathrm{~kg}$ are analysed.
Figures 7(a), 7(b), and 7(c) show the responses of the longitudinal vibration acceleration during the upward and the downward process with $m$ of 350,400 , and $450 \mathrm{~kg}$, respectively. Contrary to the vibration acceleration response in the uplink and downlink processes, the amplitude of the acceleration response of the longitudinal vibration becomes increasingly small regardless of the direction of elevator movement. In other words, with a large mass of elevator car, the longitudinal vibration of the elevator is weak. Therefore, in the tensile 


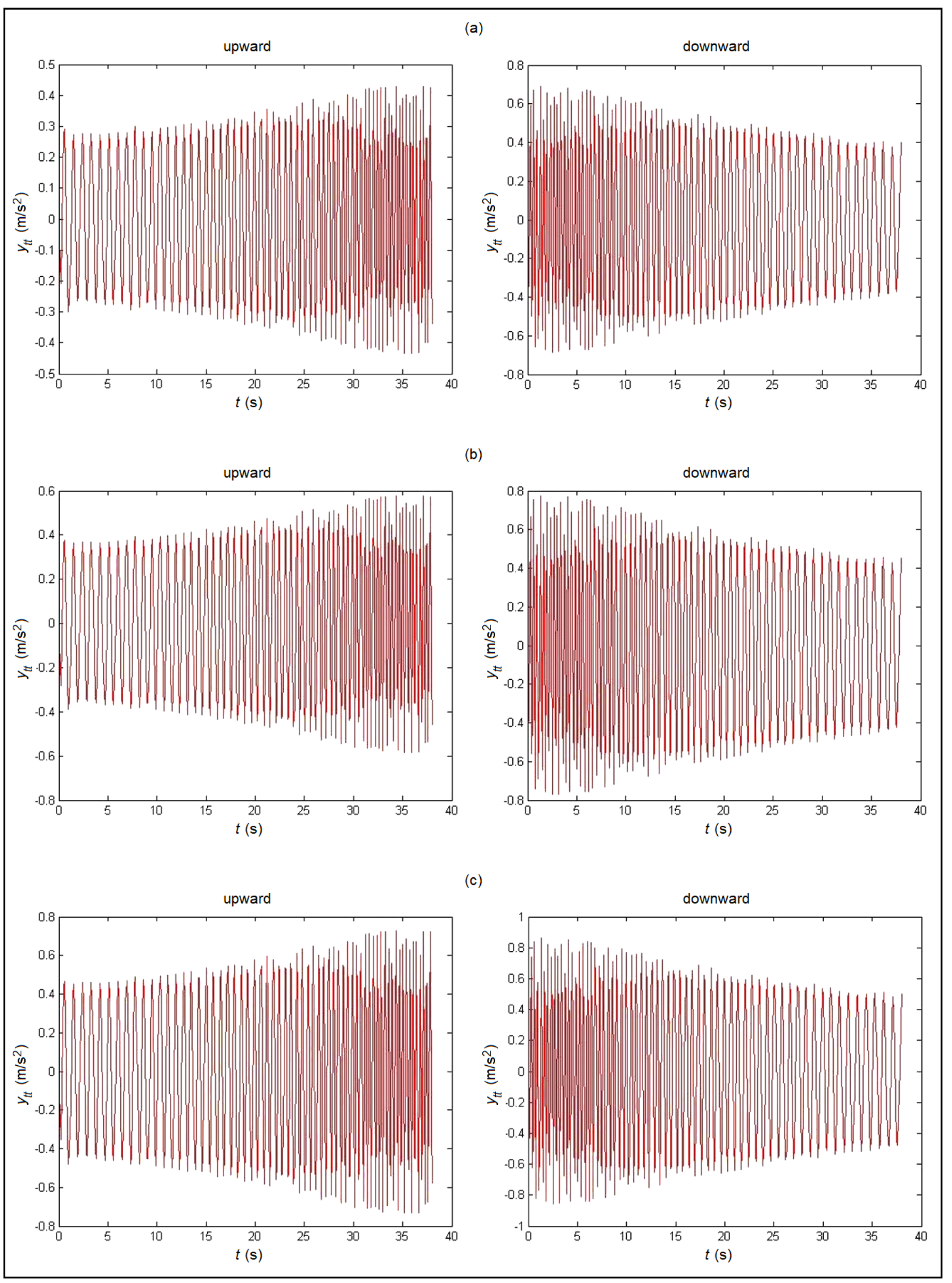

Figure 6. Effect of tensile force on longitudinal vibration of the elevator. 


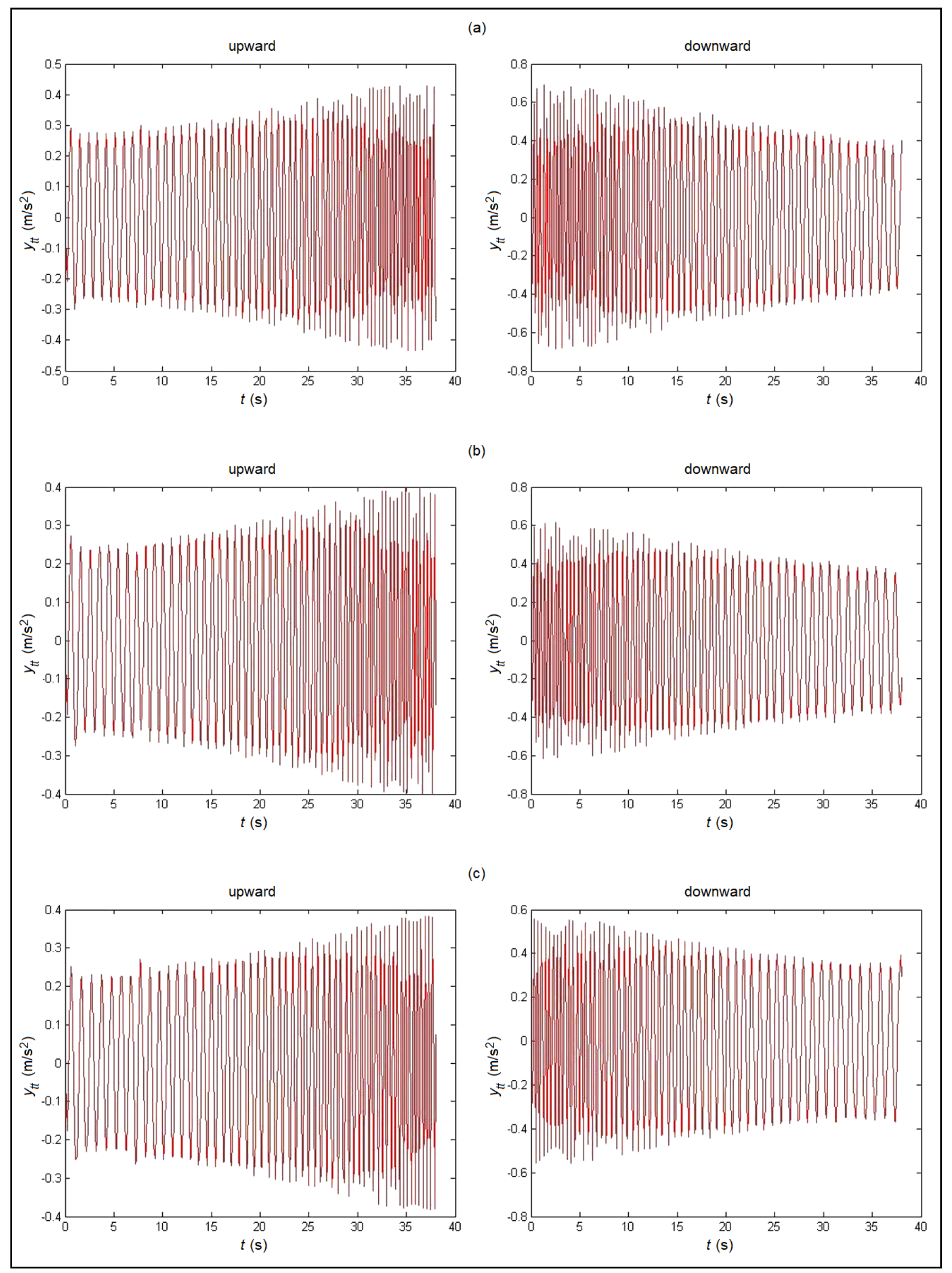

Figure 7. Effect of the lifting quality of a single rope on the longitudinal vibration of the elevator. 
range of the traction rope, the longitudinal vibration of the elevator can be reduced by appropriately increasing the weight of the car during elevator design.

\section{CONCLUSIONS}

1. Aiming at the nonlinear time-varying characteristics of the traction rope of the high-speed traction elevator hoisting system, considering the weight of the compensation rope and the tension force of the tensioning device, through studying the longitudinal vibration of the lifting system of the high-speed traction elevator, the time-varying dynamic model of the longitudinal vibration of high-speed traction elevator systems with variable mass, variable damping, and variable stiffness is established by means of the energy method and Hamliton principle.

2. The ideal operating state of the elevator, as fitted into the quintic polynomial, was utilized as an input motion parameter for simulating the longitudinal vibration time-varying dynamic response of the lifting system. Following this, the simulation analysis of the nonlinear time-varying dynamic equation of the high-speed elevator lifting system was conducted using the precise integration method. It is observed that, during operation, as the length of the hoist rope increases, the acceleration of the longitudinal vibration of the elevator increases too. As the tensile force, provided by the tensioning device, increases, the acceleration decreases.

3. This study establishes a time-varying dynamic model of longitudinal vibration in a high-speed elevator and analyses the dynamic characteristics. This work further improves the longitudinal vibration dynamic model, provides a certain reference for the study of the dynamic characteristics of the elevator lifting system and the control of the elevator vibration. This study also offers guiding significance for the design of elevator systems.

\section{ACKNOWLEDGEMENTS}

This research was supported by the Shandong Province Natural Science Foundation, China (Grant No. ZR2017MEE049), the Introduce urgently needed talents project for the western economic uplift belt and the key areas of poverty alleviation and development in Shandong Province.

\section{REFERENCES}

1 Du, X. Q., Mei, D. Q., and Chen, Z. Time-varying element model of high-speed traction elevator and its horizontal vibration response analysis, Journal of Zhejiang University (Engineering Science), 43 (1), 148-152, (2009). https://dx.doi.org/10.3785/j.issn.1008-973X.2009.01.029

2 Bao, J. H. Dynamics Modeling and Vibration Control of High-Speed Elevator Hoisting System, Shanghai JiaoTong University, (2014).

3 Mei, D. Q., Du, X. Q., and Chen, Z. Optimization of dynamic parameters for a traction-type passenger elevator using a dynamic byte coding genetic algorithm, Proceedings of the Institution of Mechanical Engineers, Part C: Journal of Mechanical Engineering Science, 223 (3) , 595-605, (2009). https://dx.doi.org/10.1243/09544062JMES1149

${ }^{4}$ Feng, W. Z., Cao, S. Q., and Hu, C. Study on frequency reliability sensitivity analysis and robust design for vertical vibration of the elevator system, Journal of Vibration, Measurement \& Diagnosis, 34 (4), 630635, (2016). https://dx.doi.org/10.16450/j.cnki.issn.10046801.2016.04.003

5 Chang, N., He, W., and Li, Z. H. Analysis of vertical vibration reduction strategy for elevator system, Noise and Vibration Control, 37 (2), 117-37, (2017). https://dx.doi.org/10.3969/j.issn.1006-1355.2017.02.024

$6 \mathrm{Wu}$, H., Ye, W. H., Shen, Y., and Tang, Z. R. Vertical vibration modeling and experimental analysis of high speed elevator, Machinery, 51 (584), 19-22, (2013). https://dx.doi.org/10.3969/j.issn.1000-4998.2013.04.007

7 Wu, L. M., Gong, Y. Y., and Xue, F. Dynamic characteristics analysis of vertical vibration of haulage type elevator mechanical system, Machinery Design \& Manufacture, 10, 16-18, (2007). https://dx.doi.org/10.3969/j.issn.10013997.2007.10.007

${ }^{8}$ Feng, W. Z., Cao, S. Q., Zhao, F., Hu, C., and Liu, W. B. Study on resonance failures sensitivity for elevator system, Journal of Vibration and Shock, 34 (1), 165-170, (2015). https://dx.doi.org/10.13465/J.CNKI.JVS.2015.01.029

${ }^{9}$ Lin, Y., Liu, Y. B., Wu, H., and Huang, Y. Z. Dynamic performance analysis and kinetic parameters optimization of high-speed elevator based on VPT, Journal of Vibration, Measurement \& Diagnosis, 35 (1), 150155, (2015). https://dx.doi.org/10.16450/j.cnki.issn.10046801.2015.01.026

10 Zhang, P., Zhu, C. M., Zhang, L. J. Analyses of longitudinal vibration and energetics on flexible hoisting systems with arbitrarily varying length, Journal of Shanghai Jiaotong University, 42 (3), 480-488, (2008). https://dx.doi.org/10.3321/j.issn:1006-2467.2008.03.027

11 Bao, J. H., Zhang, P., and Zhu, C. M. Dynamic analysis of flexible hoisting rope with time-varying length, International Applied Mechanics, 51 (6), 710-720, (2015). https://dx.doi.org/10.1007/s10778-015-0729-z

12 Bao, J. H., Zhang, P., and Zhu, C. M. Transverse vibration of flexible hoisting rope with time-varying length, Journal of Mechanical Science and Technology, 28 (2), 47-466, (2014). https://dx.doi.org/10.1007/s12206-013-1110-y

13 Bao, J. H., Zhang, P., and Zhu, C. M. Longitudinal vibration of rope hoisting systems with time-varying length, Journal of Vibration and Shock, 15, 173-177, (2013). https://dx.doi.org/10.3969/j.issn.1000-3835.2013.15.031

14 Zhong, W. X. Precise computation for transient analysis, Chinese Journal of Computational Mechanics, 12 (1), 1-6, (1995). https://dx.doi.org/10.7511/jslx19951001

15 Deng, Z. C., Zheng, H. J., Zhao, Y. L., and Zhong, W. X. On computation of dynamic properties for deploying cantilever beam based on precision integration method, Journal of Astronautics, 22 (6), 110-113, (2001). https://dx.doi.org/10.3321/j.issn:1000-1328.2001.06.018

16 Lee, S. Y., and M. Lee. A new wave technique for free vibration of a string with time-varying length, Journal of Applied Mechanics, 69 (1), 83-87, (2002). https://dx.doi.org/10.1115/1.1427337 